\section{Getting the bird}

BIRD attack on milk bottles delivered to the doorstep is an important risk factor for infection with Campylobacter jejuni, an enteric bacterial pathogen, according to a recent case-control study by J.P. Southern et al. published in The Lancet $(336,1425-1427 ; 1990)$. The study, which began after a reported four-fold increase in Campylobactercases in the Bridgend area of south Wales last May, implicates magpies and jackdaws. The authors report a strong correlation between bird attack on bottles and incidence of human illness; although they did not carry out a microbiological investigation, Campylobacter have previously been isolated from both species of bird.

\section{Hubble trouble}

IN trenchant style (beginning with a quote from the Federalist Papers on the capacity of wise men to indulge in controversy), A. Sandage and G. A. Tammann, two veterans of the search for Hubble's constant, deliver their latest findings in the Astrophysical Journal (365, 1-12, 1990). In our immediate vicinity, Hubble's constant $\left(H_{0}\right)$, the rate at which the galaxies recede, is widely believed to be $50 \mathrm{~km}$ per second per megaparsec, at the low end of the empirical spectrum of values, which stretches up to $100 \mathrm{~km} \mathrm{~s}^{-1} \mathrm{Mpc}^{-1}$. But our local group of galaxies has a peculiar velocity towards the giant Virgo cluster, which contaminates extrapolations of $H_{0}$ to the much-desired cosmic value. Sandage and Tammann compare the Virgo cluster directly with more remote clusters to establish the 'true' recession velocity of Virgo. They deduce that $H_{0}=$ 50 holds true cosmologically as well as locally - a result that will surprise neither their supporters nor members of the $H_{0}=100$ gang.

\section{Mating games}

Work by C. L. Jackson and L. H. Hartwell shows that cells of the budding yeast Saccharomyces cerevisiae choose their mating partner according to the strength of a pheromone signal. Haploid yeast cells exist in two mating types, a and $\alpha$, which can conjugate to form $\mathbf{a} / \alpha$ diploids. Conjugation requires arrest of the mitotic cycle, activation of several genes essential for cell fusion and karyogamy, and a number of morphological changes that align the two cells. These processes are all initiated by the binding of a pheromone to specific cell-surface receptors. Writing in Molecular and Cellular Biology $(\mathbf{1 0}, 2202-2213$; 1990) and Cell (63, 1039-1051; 1990), Jackson and Hartwell show that individual $\mathbf{a}$ and $\alpha$ cells select as a partner the cell secreting the most pheromone. Perhaps other eukaryotes respond to external signals in the same way to cause directed cell movement or growth.

\section{Thereby hangs a tail}

\section{Chris Paul}

WiTH our anthropocentric view of evolutionary 'progress', we naturally seek our most distant ancestors among the invertebrates. There is a consensus that echinoderms (sea urchins, starfish and their allies) are our closest relatives among non-chordate phyla, but there has been considerable resistance to the only consistent attempt to develop a detailed account of echinoderm-chordate relationships. This is the work of R. P. S. porous structure called stereom and, typically, pentameral symmetry; and, finally, the carpoids (Fig. $1 d-f$ ), bizarre, extinct creatures with echinoderm-like stereom plates, but no trace of pentamerism. There is general agreement that carpoids form an evolutionary line distinct from that of typical pentameral echinoderms ${ }^{3 .+}$. But according to conventional wisdom (Fig. 2a) invertebrate chordates are more closely related to vertebrates than echi-
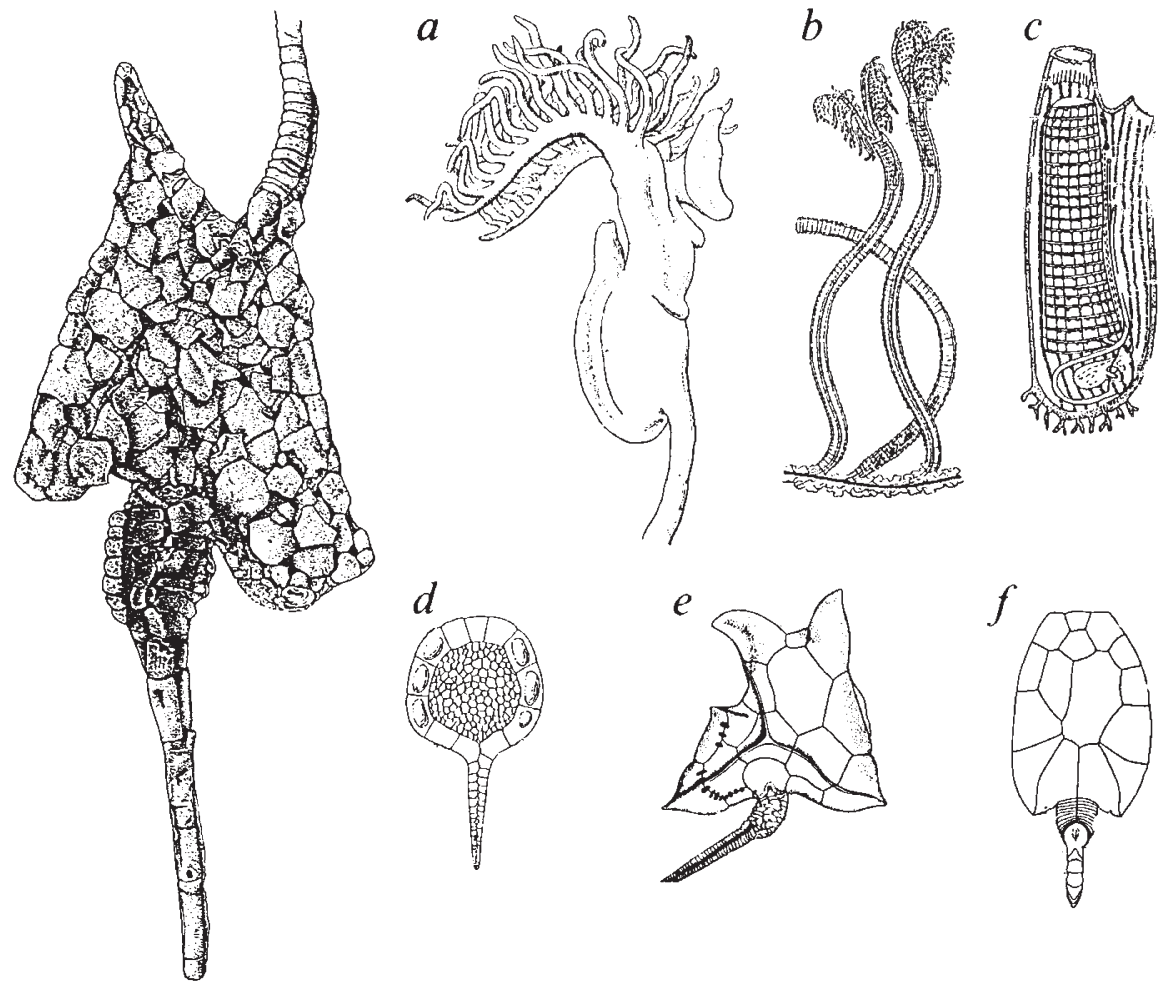

FIG. 1. Left, Dendrocystoides scoticus in dorsal view, the feeding arm being the longer structure at the top, the tail being below. Right, invertebrate chordates $(a-c)$ and carpoids $(d-f) . a, b$, Rhabdopleura, a hemichordate ( $a$, an individual, $b$, the colony); $c$, Ciona, a solitary urochordate; $d$, Trochocystites, a cinctan; e, Ceratocystis, a cornute carpoid; $f$, Mitrocystella, a mitrate.

Jefferies, whose latest contribution' deals with an obscure Palaeozoic fossil Dendrocystoides scoticus (Fig. 1). The paper depicts for the first time Jefferies's views of the relationships between all the groups of fossils that Jaekel ${ }^{2}$ first distinguished as carpoids (and which most palaeontologists regard as aberrant echinoderms). It also confounds the alternative hypothesis about the carpoid 'feeding tail' favoured by some students of fossil echinoderms.

The main controversy involves four groups of organisms. The vertebrates, a subgroup of chordates characterized by possession of a backbone made of vertebrae; the 'invertebrate chordates' (Fig. $(a-c)$, a morphologically diverse group of living organisms that at some stage in their life cycle possess a notochord which in vertebrates acts as the template for the backbone: the echinoderms, which lack a notochord but possess calcite plates with a noderms because they possess a notochord, and carpoids are echinoderms because they possess stereom plates. Reduced to its simplest, Jefferics"s view is that the phylogenetic relationships are more complex than that, with some carpoids being more closely related to vertebrates than to some invertebrate chordates (Fig. $2 b$ ).

During their ontogeny living echinoderms undergo remarkable, asymmetrical developments. Starting with an elongate, bilaterally symmetrical, motile larva with three pairs of coelomic cavities that are very similar to the larvae of some hemichordates, they develop extensive organ systems from the left-hand coeloms, whereas those on the right atrophy. Jefferies explains this by suggesting that an ancestor similar to the colonial hemichordate Cephalodiscus lay down on its right-hand side, a condition he calls dexio- 